\title{
A meta-analysis and systematic review of propofol on liver ischemia-reperfusion injury protection during hepatocellular carcinoma anesthesia surgery
}

\author{
Changsheng Feng, Duo Qian, Changlin Chen
}

Department of Anesthesiology, Affiliated Hospital of North Sichuan Medical College, Nanchong, China

Contributions: (I) Conception and design: C Feng, D Qian; (II) Administrative support: C Chen; (III) Provision of study materials or patients: C Feng, D Qian; (IV) Collection and assembly of data: All authors; (V) Data analysis and interpretation: C Feng, C Chen; (VI) Manuscript writing: All authors; (VII) Final approval of manuscript: All authors.

Correspondence to: Changsheng Feng. 63 Cultural Road, Shunqing District, Nanchong, China. Email: boxfchsh@126.com.

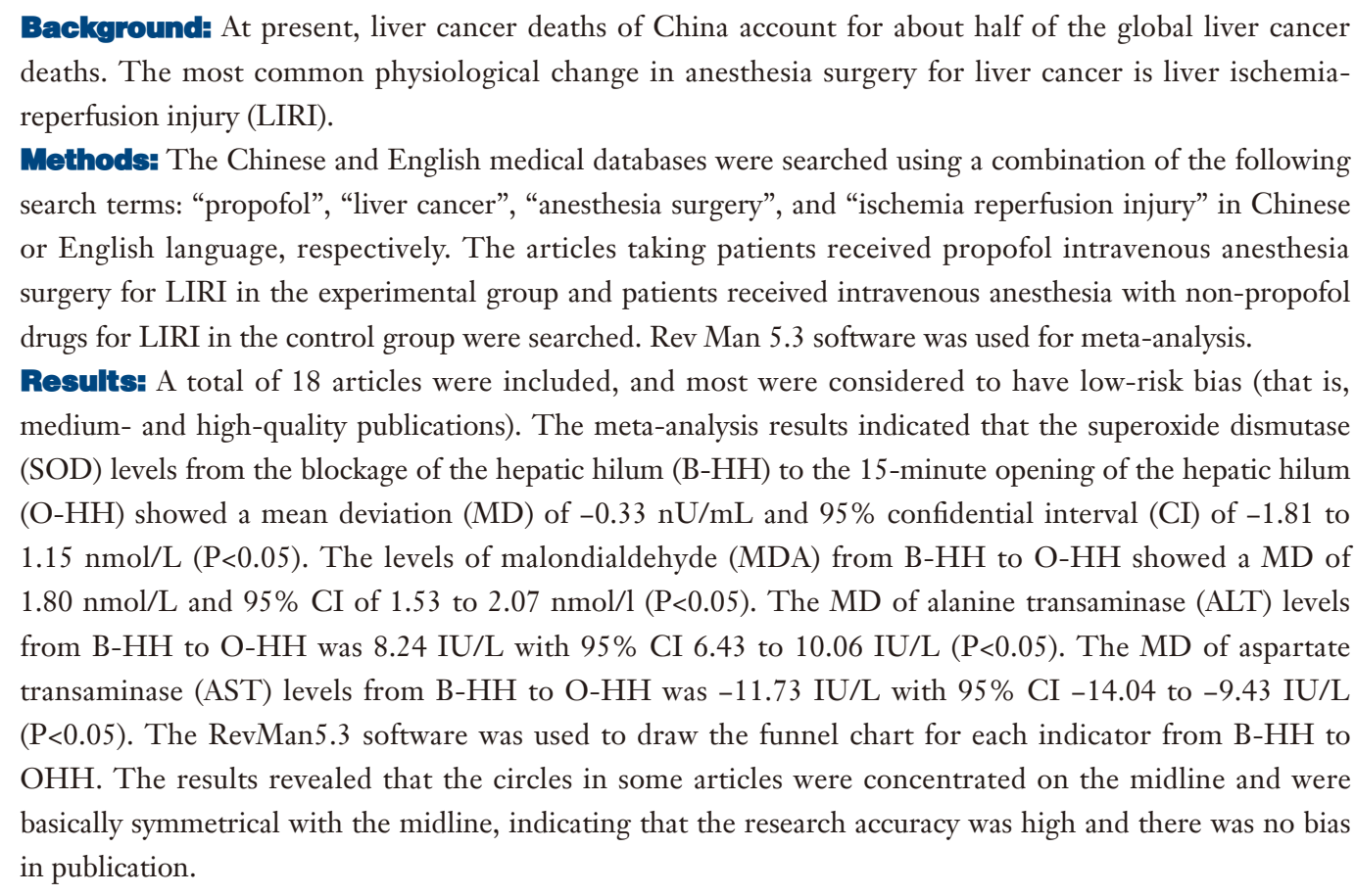

Discussion: This meta-analysis confirmed that propofol exerted a protective effect on LIRI during anesthesia surgery with hepatic hilar blockade.

Keywords: Propofol; liver cancer; anesthesia surgery; ischemia reperfusion injury; protective effect

Submitted Apr 16, 2021. Accepted for publication Jun 01, 2021.

doi: 10.21037/apm-21-1242

View this article at: https://dx.doi.org/10.21037/apm-21-1242

\section{Introduction}

Liver is one of the important organs for human metabolism and it is one component the blood transport system. With development and change of living ways of people, the incidence of liver disease has continued to increase (1). Statistics shows that about 360 million people worldwide suffer from diseases caused by liver dysfunction. There are an estimated 1.1 million newly diagnosed liver cancer 
patients each year, and about $25 \%$ are from China (2). Liver cancer is one of the most common malignant tumors in China, with a high mortality rate, ranking the third among malignant tumors. Hepatocellular carcinomas proliferate rapidly, deteriorate easily, have high recurrence rates, and high incidences of metastasis. The symptoms are generally not obvious in the early stages, and at diagnosis, most patients are in the middle to late stages of liver cancer. At present, the mainstream clinical treatments for liver cancer include interventional therapy, surgical treatment, radiotherapy, and liver transplantation. The most common treatment regimen refers to the liver resection surgery under anesthesia (3). During the liver resection surgery, the site of surgical resection is prone to bleeding due to the complicated blood supply of the liver. When the liver regains the blood supply, not only the liver function can't be restored, but the dysfunction of metabolism is aggravated. This phenomenon is called LIRI.

During liver surgery under anesthesia, different anesthetic drugs and methods are used, all of which have certain impacts on liver function. As an intravenous anesthetic, propofol induces anesthesia smoothly and quickly. Its advantages include ease of control of the anesthetic effect, rapid metabolism in the body, no drug accumulation, and no toxic side effects, and is thus widely used in clinical practice (4). Wei et al. [2019] (5) pointed out that propofol had a protective effect on LIRI in rats. Research by Li et al. [2019] (6) revealed that propofol had an antioxidant effect on cardiac ischemia-reperfusion injury in rats. However, whether the above studies have a protective effect on clinical LIRI still needs a large number of studies to prove. During LIRI, the amount of oxygen free radicals in the serum increases, and this can be measured by the levels of superoxide dismutase (SOD) and malondialdehyde (MDA) (7). The increased activity of superoxide dismutase can indicate that the body's ability to decompose free radicals is enhanced. When LRIRI occurs, its activity decreases and the decomposition level decreases. MDA can reflect the degree of lipid peroxidation, and its value increases during ischemia-reperfusion injury. Aminotransferase (ALT) and aspartate transaminase (AST) levels can be used as indicators of liver cell damage (8).

The innovation of this study lied in the use of Metaanalysis to compare the serum levels of superoxide dismutase and malondialdehyde in patients undergoing intravenous anesthesia with propofol and intravenous anesthesia without propofol, aiming to explore whether propofol has a protective effect on patients with LIRI during liver anesthesia surgery, and to provide a scientific reference for the selection of a reasonable anesthesia plan for clinical liver cancer anesthesia surgery.

We present the following article in accordance with the PRISMA reporting checklist (available at https://dx.doi. org/10.21037/apm-21-1242).

\section{Methods}

\section{Retrieval of related articles}

A search was performed using PubMed, Medline, Embase, China Biomedical Literature (CBL) database, China National Knowledge Network (CNKI) database, Wanfang database, VIP database, and Google Scholar. The retrieval time was from the establishment of the database to December 15, 2020. Relevant articles were selected using the compound Boolean logic retrieval method. The Chinese database was searched using a combination of the following term: "propofol", "liver cancer", "anesthesia surgery", and "ischemia reperfusion injury". The English databases were searched using a combination of "propofol", "liver cancer", "anesthesia surgery", and "ischemia reperfusion injury" as the search terms. The quality of the literature was evaluated using the Rev Man 5.3 software provided by the Cochrane system.

The search terms were combined freely over multiple searches to confirm the retrieved articles, which were further verified using the search engine. The latest research progress was obtained after consultation with experts and researchers in the field.

\section{Inclusion and exclusion criteria of the articles}

The following inclusion criteria were applied: articles related to anesthesia surgery for the exploration of the protective effect of propofol on ischemic reperfusion injury; subjects of the articles were older than 18 years; articles with pathological control analysis with reliable $95 \%$ confidence intervals (CIs); articles where the experimental group was patients who underwent general anesthesia by intravenous injection of propofol; and articles in which the diagnosis of liver cancer was based on the standards of the World Health Organization (WHO).

The following exclusion criteria were applied: articles with repeated research subjects; duplicate articles; articles on animal experiments or liver tumor cell experiments; articles with unavailable or incomplete data after contacting the 
Table 1 The four levels in the GRADE quality classification scale

\begin{tabular}{ll}
\hline Quality level & Definition \\
\hline Extremely low & The estimated effect was not believable; and the estimated effect was completely different from the actual value \\
Low & The estimated effect was partially believed; and the estimated effect was completely different from the actual value \\
Medium & The estimated effect was believed to a certain degree; and it approached the actual value \\
High & The estimated effect can be believed, because it was the same as the actual value \\
\hline
\end{tabular}

original author; and articles not related to anesthetic surgery.

Two senior experts independently screened the titles, abstracts, and full texts of the articles, and 3 preliminary operations were performance before the final screening. Any inconsistencies between the two experts were resolved by discussion or arbitration with a third expert.

\section{Evaluation indicators}

The levels of SOD and MDA were detected at the time of blockage of the hepatic hilum (B-HH) and at the time of opening of the hepatic hilum (O-HH). The levels of ALT and AST at B-HH and $\mathrm{O}-\mathrm{HH}$ were also assessed.

\section{Data extraction}

The data was extracted from the included articles by two experts independently using a unified Excel table, and the data should be extracted for 3 times as the trails before the final extraction. Any inconsistencies between the two experts were resolved by discussion or arbitration with a third expert. The data extracted included the following characteristics: title, first author, year of publication; name of publication; basic information of the research subjects including average age, gender, and body mass index (BMI); the anesthesia methods of the experimental group and the control group, and the use of propofol; and the source, sample size, and outcome indicators of the propofol general anesthesia group and the control group.

\section{Risk of bias assessment}

Two researchers were invited to assessment the risk of bias. Any inconsistencies between the two experts were resolved by discussion or arbitration with a third expert. In this study, the Cochrane Collaboration for "bias risk assessment" for randomized controlled trials (RCTs) was used. The evaluation criteria included random allocation method, blind method, allocation plan hiding, completeness of data results, and research results. Articles were classified as "high risk bias", "low risk bias", or "unclear risk bias" papers.

\section{Quality assessment}

Two experts were invited to assess the quality of included articles. If there was any disagreement between the two experts, it can be determined by discussion or arbitration of the third expert. The GRADE standard in the Cochrane Collaboration was used for quality classification (Table 1). Selected articles with a score of 7 and above were considered high quality (low risk bias); articles with a score of 1 or less were considered low quality (high risk bias); and articles with a score of 2-6 were considered medium quality (unclear risk bias).

\section{Statistical analysis}

All data was statistically analyzed with the StataSE12.0 software. Odds ratio (OR) and 95\% CI were evaluated for SOD, MDA, ALT, and AST levels in patients with and without propofol intravenous anesthesia. The bias risk assessment charts were drawn by Rev Man 5.3 software. Each effect was expressed using a 95\% CI. When $\mathrm{P}>0.1$ and $\mathrm{I}^{2} \leq 50 \%$, the fixed effects model (FEM) was used for metaanalysis. When $\mathrm{P}<0.1$ and $\mathrm{I}^{2}>50 \%$, the random effects model (REM) was used for meta-analysis.

\section{Results}

\section{Search results and basic characteristics of the included articles}

Out of the 2,289 articles obtained after the preliminary search, 2,237 articles were excluded through review of the abstracts and titles, 34 articles were eliminated through reading the full text, and 18 articles were finally included for this meta-analysis. The main reasons for exclusion were as follows: 1,136 articles had duplicate research subjects, 


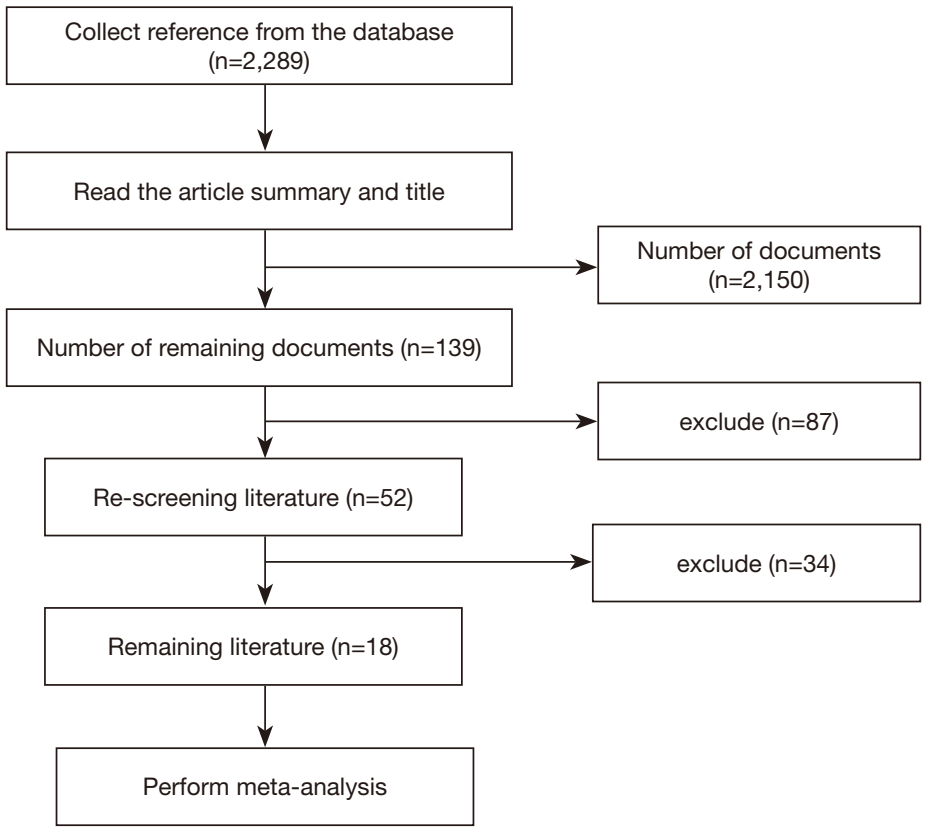

Figure 1 A flow chart showing the article retrieval process.

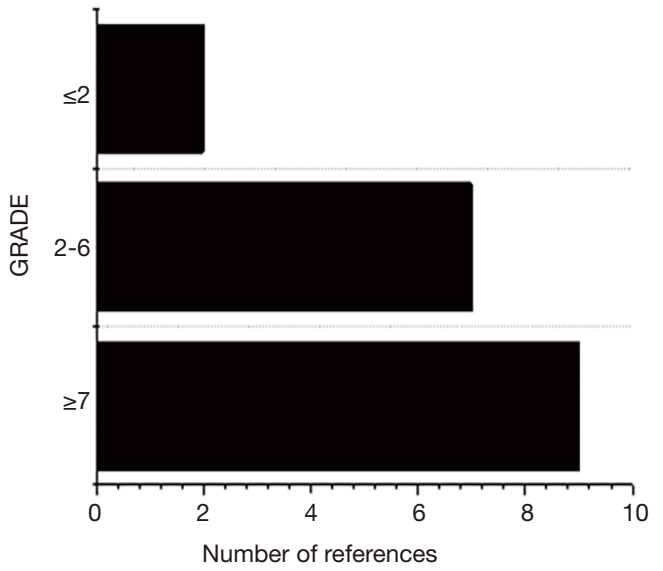

Figure 2 The quality classification results of the included literature based on GRADE.

294 articles did not perform case-control analyses, the research subjects of 398 articles did not undergo anesthesia surgery for liver cancer, and 443 articles had unavailable research information (Figure 1). Figure 2 shows the quality classification results based on GRADE. There were 9 articles with a score of 7 or above, 7 articles with a score of $2-6$, and 2 articles with a score of 2 .

A total of 18 articles (containing a total of 985 patient cases) satisfied the inclusion criteria, including 14 articles that included a blank control group, 2 articles included an enflurane control group, and 2 articles included an isoflurane control group. All 18 publications were small-sample studies, with the sample size ranging from 14 to 164 , and all research subjects were over the age of 18 years. A total of 15 articles described in detail the gender, age, BMI, and time of hepatic portal blockade of patients the experimental group and the control group. The basic characteristics of the research subjects are shown in Table 2.

\section{Risk bias assessment of the included articles}

Figures 3 and 4 show the results of multiple risk bias evaluations of the included documents. Among the 18 RCTs in this study, 2 trials $(9,10)$ described the correct random allocation method, and only one (11) described the correct random allocation method and the hidden allocation plan in detail. Only one (12) article used the blinding method. However, the measurement indicators in this study were laboratory indicators determined by the computer, hence, it can be considered that all articles used the blinding method correctly.

\section{Comparison on SOD levels}

A total of 7 articles analyzed the levels of plasma SOD in 
Table 2 The basic characteristics of the research subjects in the included literature

\begin{tabular}{|c|c|c|c|c|c|c|c|c|}
\hline First author & $\begin{array}{c}\text { Year of } \\
\text { publication }\end{array}$ & $\begin{array}{l}\text { Number of } \\
\text { patients }\end{array}$ & Country & Age (years) & Males & Females & BMI $\left(\mathrm{m}^{2} / \mathrm{kg}\right)$ & $\begin{array}{c}\text { Course of } \\
\text { disease (years) }\end{array}$ \\
\hline Hausburg MA & 2020 & 58 & Brazil & $40.4 \pm 9.7$ & 28 & 30 & $28.7 \pm 5.0$ & $5.0 \pm 2.1$ \\
\hline XuF & 2018 & 95 & China & $61.6 \pm 9.9$ & 45 & 50 & $30.9 \pm 3.9$ & $3.2 \pm 2.1$ \\
\hline Yan HJ & 2019 & 84 & China & $42.8 \pm 8.5$ & 45 & 39 & $30.6 \pm 5.3$ & $5 \pm 1.6$ \\
\hline Li YM & 2019 & 49 & China & $25.7 \pm 6.5$ & 25 & 24 & $22.6 \pm 2.4$ & $4.6 \pm 2.8$ \\
\hline Kim D & 2020 & 56 & Russia & $63.1 \pm 7.3$ & 26 & 30 & - & $5.3 \pm 1.1$ \\
\hline Hao W & 2017 & 158 & Iraq & - & - & - & - & - \\
\hline
\end{tabular}

BMI, body mass index.

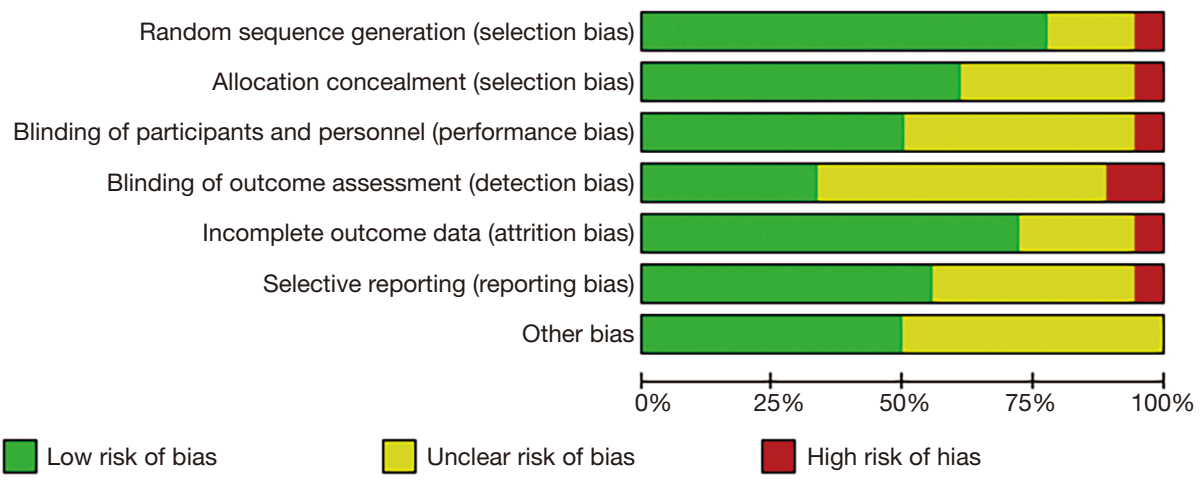

Figure 3 Assessment of risk bias of the included articles.

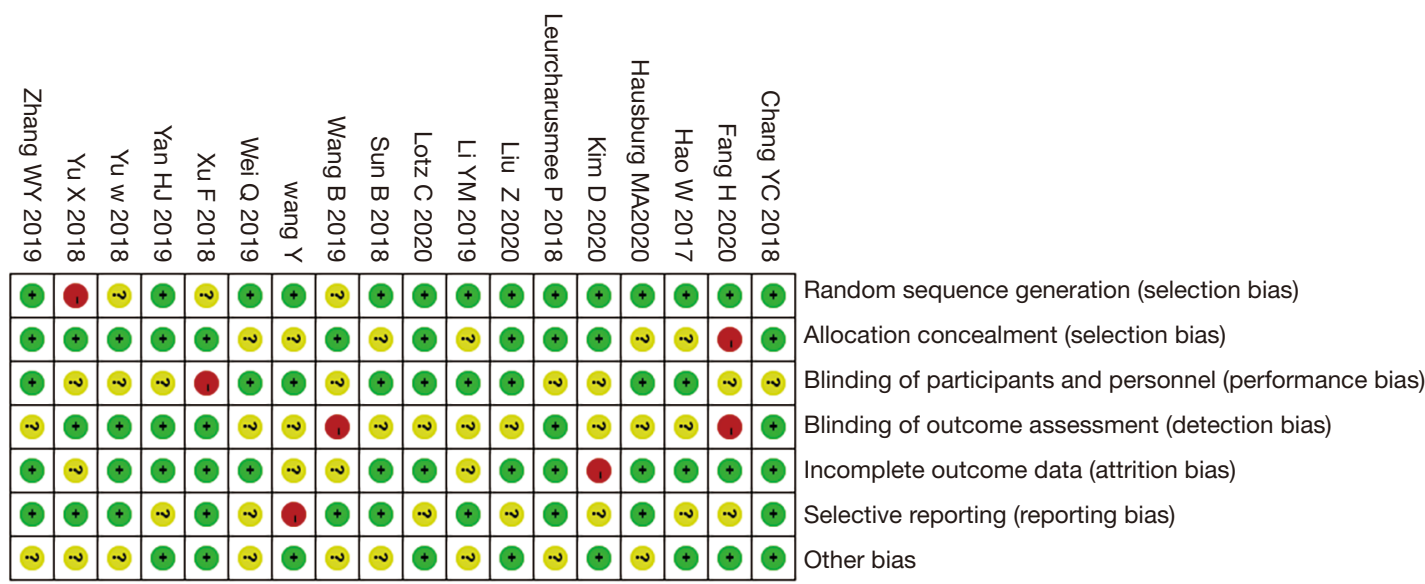

Figure 4 Multiple studies in the literature correspond to the multiple risk bias evaluation results. 


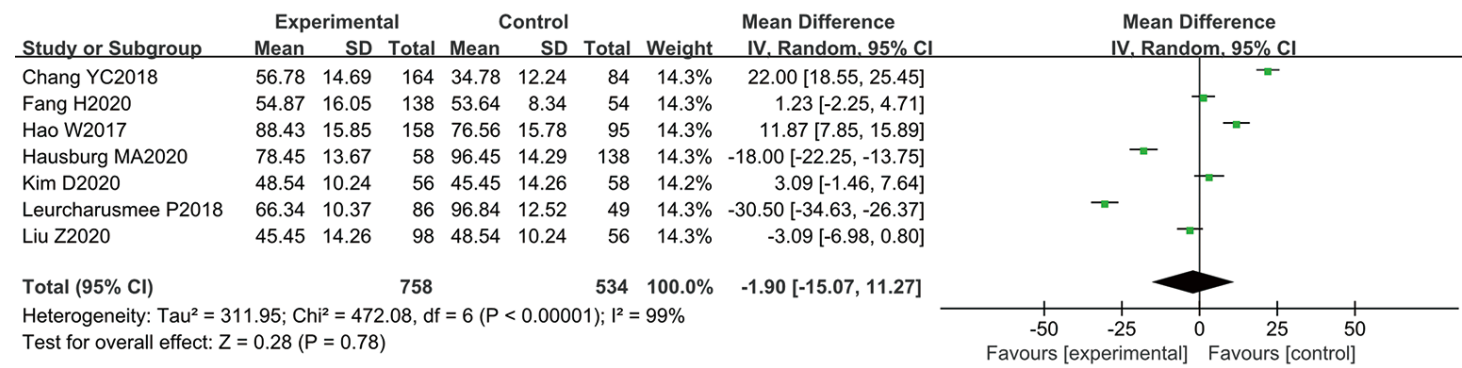

Figure 5 Superoxide dismutase (SOD) levels in patients from blockage of the hepatic hilum (B-HH) to time of opening of the hepatic hilum $(\mathrm{O}-\mathrm{HH})$.

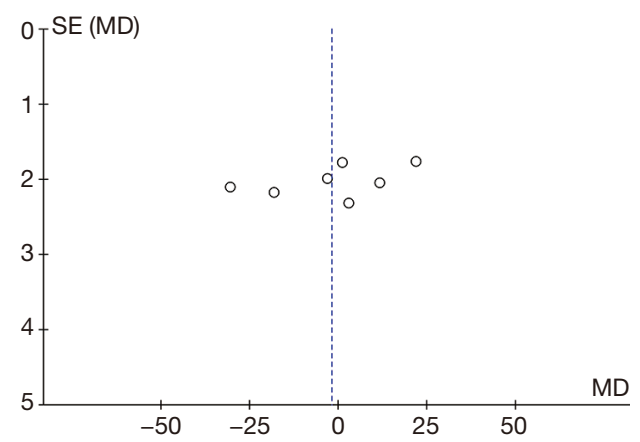

Figure 6 The funnel chart of superoxide dismutase (SOD) levels from blockage of the hepatic hilum $(\mathrm{B}-\mathrm{HH})$ to time of opening of the hepatic hilum $(\mathrm{O}-\mathrm{HH})$.

patients from $\mathrm{B}-\mathrm{HH}$ to $\mathrm{O}-\mathrm{HH}$. There were 1,292 cases in total, including 758 cases in the experimental group, and 534 cases in the control group. The heterogeneity test showed $\mathrm{I}^{2}=99 \%(\mathrm{P}<0.00001)$. The research results of Chang et al. [2018] (8) accounted for the highest percentage of the final combined results (18.4\%), followed by the results of Hao et al. [2017] (9). In addition, the horizontal lines of the $95 \%$ CI of some studies were on the left of the invalid vertical line, a few research horizontal lines crossed the invalid vertical line, and the horizontal line of some $95 \%$ CI were on the right side of the invalid vertical line. Analysis using the REM (Figure 5) showed that the difference between the experimental group and the control group was statistically significant, with a MD of -0.33 and $95 \%$ CI -1.81 to $1.15(\mathrm{P}<0.05)$.

Figure 6 shows the funnel chart of SOD levels from $\mathrm{B}-\mathrm{HH}$ to $\mathrm{O}-\mathrm{HH}$. The circles in some studies were basically symmetrical to the midline, suggesting that the research accuracy was high and there was no bias in publication.

\section{Comparison on MDA level}

A total of 7 articles analyzed the levels of MDA in the plasma of patients from B-HH to O-HH. There were 643 cases in total, including 442 cases in the experimental group, and 201 cases in the control group. The heterogeneity test showed $\mathrm{I}^{2}=97 \%(\mathrm{P}<0.00001)$, indicating that the heterogeneity among the experimental groups was large. In most studies, the horizontal line of the $95 \%$ CI fell on the right side of the invalid vertical line, a few studies crossed the invalid vertical line, and the horizontal line of the individual 95\% CI fell on the left side of the invalid vertical line. The REM analysis results revealed that the difference between the two groups was statistically significant. The MD was 1.80 , and the $95 \%$ CI was 1.53 to 2.07$)(\mathrm{P}<0.05$; Figure 7$)$.

The funnel chart of MDA levels from B-HH to O-HH is shown in Figure 8. The circles in some studies were concentrated on the midline and were basically symmetrical to the midline, suggesting that the research accuracy was high and there was no bias in publication.

\section{Comparison on ALT}

A total of 10 articles explored the plasma ALT levels in patients from B-HH to O-HH. There were a total of 762 cases, including 358 cases in the experimental group and 404 cases in the control group. The heterogeneity test showed $\mathrm{I}^{2}=95 \%(\mathrm{P}<0.00001)$, indicating that the heterogeneity among the experimental groups was large. In most studies, the horizontal line of the $95 \%$ CI fell on the right side of the invalid vertical line, a few studies crossed the invalid vertical line, and the horizontal line of the individual 95\% CI was on the left side of the invalid vertical line. The REM showed that the difference between the experimental group and the control group was statistically significant. The 


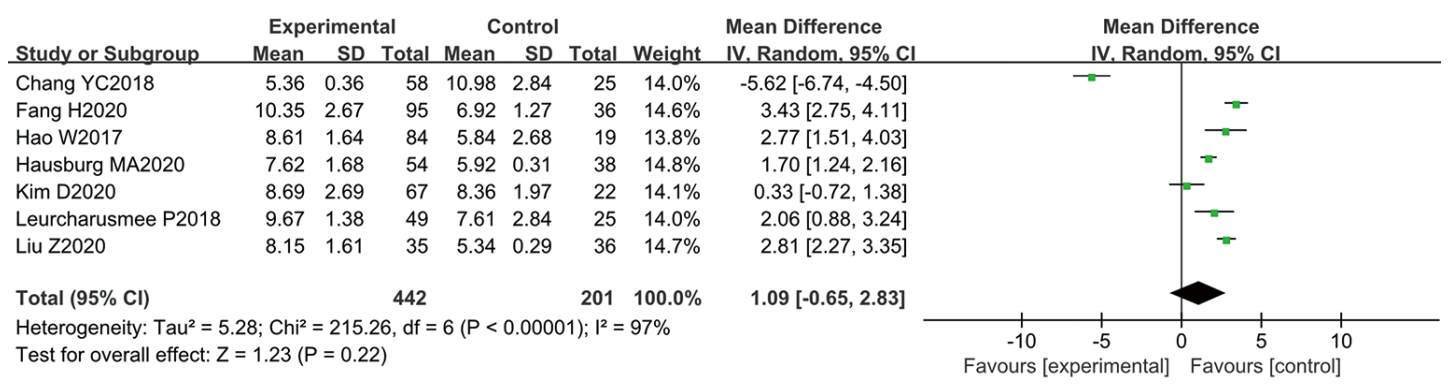

Figure 7 Malondialdehyde levels from blockage of the hepatic hilum (B-HH) to time of opening of the hepatic hilum (O-HH).

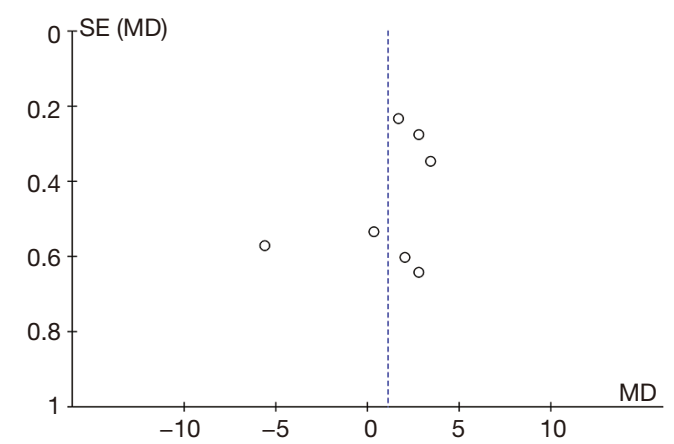

Figure 8 The funnel chart of malondialdehyde levels from blockage of the hepatic hilum $(\mathrm{B}-\mathrm{HH})$ to time of opening of the hepatic hilum $(\mathrm{O}-\mathrm{HH})$.

MD was $8.24 \mathrm{IU} / \mathrm{L}$, and the $95 \%$ CI was 6.43 to $10.06 \mathrm{IU} / \mathrm{L}$ $(\mathrm{P}<0.05$; Figure 9).

The funnel chart of the plasma ALT levels from B-HH to $\mathrm{O}-\mathrm{HH}$ is shown in Figure 10. The circles of some studies were concentrated on the midline and were symmetrical to the midline basically, suggesting that the research showed high accuracy and no bias in publication.

\section{Comparison on AST level}

A total of 10 articles assessed the plasma ALT levels in patients from $\mathrm{B}-\mathrm{HH}$ to $\mathrm{O}-\mathrm{HH}$. There were a total of 762 cases, including 358 cases in the experimental group and 404 cases in the control group. The heterogeneity test showed $\mathrm{I}^{2}=98 \%(\mathrm{P}<0.00001)$, indicating that the heterogeneity among the experimental groups was large. In most studies, the horizontal line of the $95 \%$ CI was on the left side of the invalid vertical line, a few studies crossed the invalid vertical line, and the horizontal line of the individual 95\% CI was on the right side of the invalid vertical line. The REM revealed that the difference between the experimental group and the control group was remarkable, with a $\mathrm{MD}$ of $-11.73 \mathrm{IU} / \mathrm{L}$ and $95 \% \mathrm{CI}$ of -14.04 to $-9.43 \mathrm{IU} / \mathrm{L}(\mathrm{P}<0.05$; Figure 11).

Figure 12 illustrates the funnel chart of plasma AST levels from $\mathrm{B}-\mathrm{HH}$ to $\mathrm{O}-\mathrm{HH}$. The circles in some studies were concentrated on the midline and were basically symmetrical to the midline, revealing that the research showed high accuracy and no bias in publication.

\section{Discussion}

This meta-analysis compared the protective effects of propofol and non-propofol anesthesia surgery on LIRI (13). The levels of SOD, MDA, ALT, and AST in patients who underwent intravenous anesthesia with propofol were significantly different from those in patients who underwent intravenous anesthesia without propofol (14). The SOD levels from $\mathrm{B}-\mathrm{HH}$ to $\mathrm{O}-\mathrm{HH}$ showed a $\mathrm{MD}$ of $-0.33 \mathrm{nU} / \mathrm{mL}$ with $95 \% \mathrm{CI}$ of -1.81 to $1.15 \mathrm{nmol} / \mathrm{L}(\mathrm{P}<0.05)$. The MDA levels from $\mathrm{B}-\mathrm{HH}$ to $\mathrm{O}-\mathrm{HH}$ showed a $\mathrm{MD}$ of $1.80 \mathrm{nmol} / \mathrm{L}$ and $95 \%$ CI of 1.53 to $2.07 \mathrm{nmol} / \mathrm{L}(\mathrm{P}<0.05)$. The ALT and AST levels had a MD of $8.24 \mathrm{IU} / \mathrm{L}$ and $-11.73 \mathrm{IU} / \mathrm{L}$, respectively, and the $95 \%$ CI was 6.43 to $10.06 \mathrm{IU} / \mathrm{L}$ and -14.04 to $-9.43 \mathrm{IU} / \mathrm{L}$, respectively $(\mathrm{P}<0.05)(15)$. The funnel charts of various indicators revealed that some of the circles were concentrated on the midline and were basically symmetrical with the midline, suggesting that the research accuracy was high and there was no bias in publication (16). The MDA, ALT, and AST levels in patients treated with propofol were much lower than those in patients treated with other anesthetic drugs, which the SOD levels were obviously higher. The comparisons showed statistical differences $(\mathrm{P}<0.05)$. This suggested that propofol can be used in anesthesia surgery to protect liver cells and minimize LIRI (17). However, it is noteworthy that adverse events associated with propofol were not mentioned in all the 


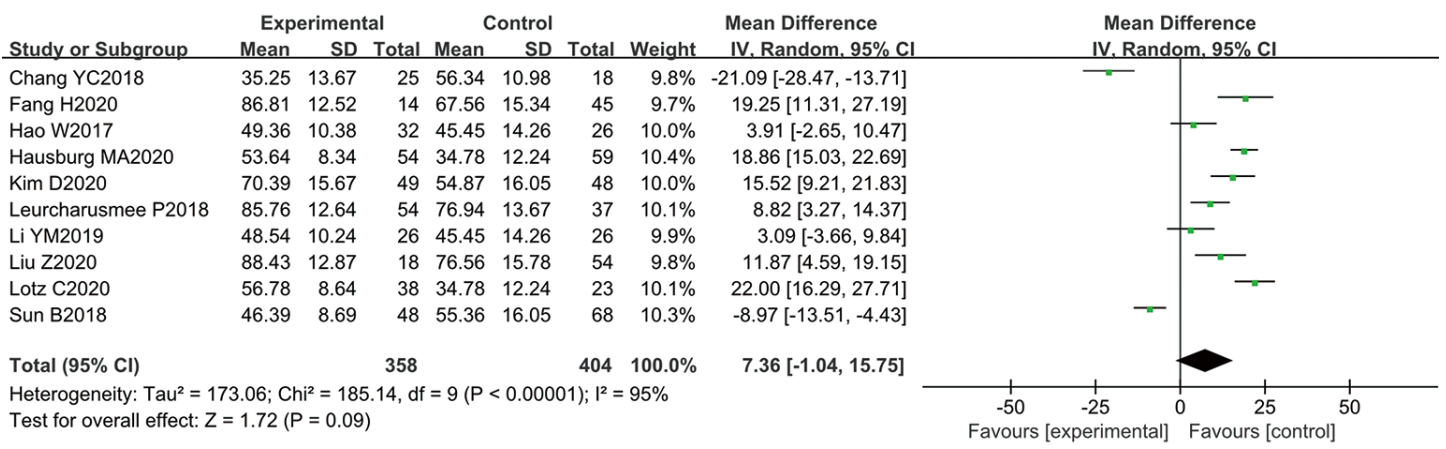

Figure 9 Aminotransferase levels from blockage of the hepatic hilum (B-HH) to time of opening of the hepatic hilum (O-HH).

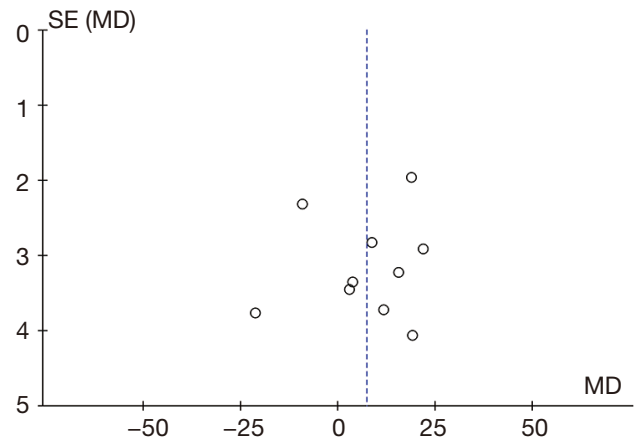

Figure 10 The funnel chart of aminotransferase levels from blockage of the hepatic hilum $(\mathrm{B}-\mathrm{HH})$ to time of opening of the hepatic hilum $(\mathrm{O}-\mathrm{HH})$.

included studies. The results of the meta-analysis of Lin et al. [2004] (18) showed that propofol had a protective effect on LIRI after liver ischemia-reperfusion by reducing the level of oxygen free radicals and inhibiting lipid peroxidation. The innovation of this study was that compared with other anesthetics, the protective effect of propofol on ischemiareperfusion was statistically significant, confirming that it had a protective effect on LIRI.

In this current investigation, most of the articles were in Chinese, and only 5 articles were in English. This could be because China has the highest incidence of liver cancer worldwide, and thus, it represents the major source of cases. Due to this, the scope of application in this study may be limited (19). The patients included in this study were mainly middle-aged and elderly patients, and propofol was used for anesthesia during hepatectomy of the hepatic port block (20). Therefore, there may be a clear distinction between minors and patients who have not undergone hepatectomy under anesthesia. The results of the GRADE quality grading in this report revealed that there were 9 articles with a score of 7 or more (high quality), 7 articles with a score of 2-6 (mediate quality), and 2 articles were low quality. The inconsistency of evaluation grades may be related to the different dosage of propofol in each group and the use of different anesthetics in the control groups. The research sample size included in this meta-analysis was small, and the number of middleand high-quality research publications was insufficient. Furthermore, the study was limited by the low quality of the research subjects and high heterogeneity. Future studies involving larger sample sizes should be conducted to further verify the conclusions of this meta-analysis.

\section{Conclusions}

In this meta-analysis, the Boolean logic retrieval method was adopted to include 18 articles that used propofol intravenous anesthesia as the experimental group and nonpropofol intravenous anesthesia as a control study. The study confirmed that propofol exerted a protective effect on LIRI during anesthesia surgery for hepatectomy with hilar blockade. 


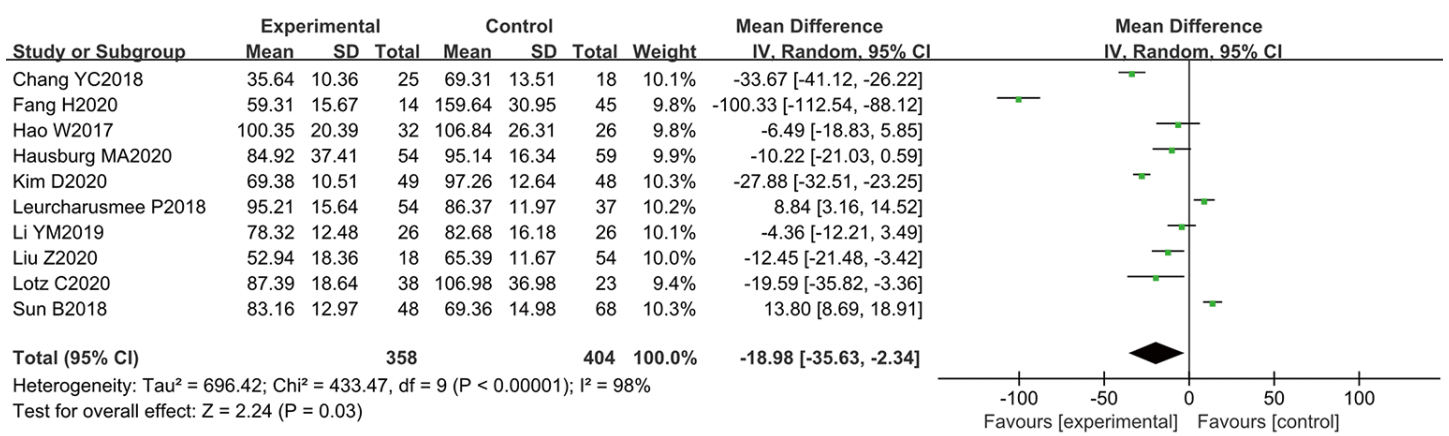

Figure 11 Aspartate transaminase levels from blockage of the hepatic hilum (B-HH) to time of opening of the hepatic hilum (O-HH).

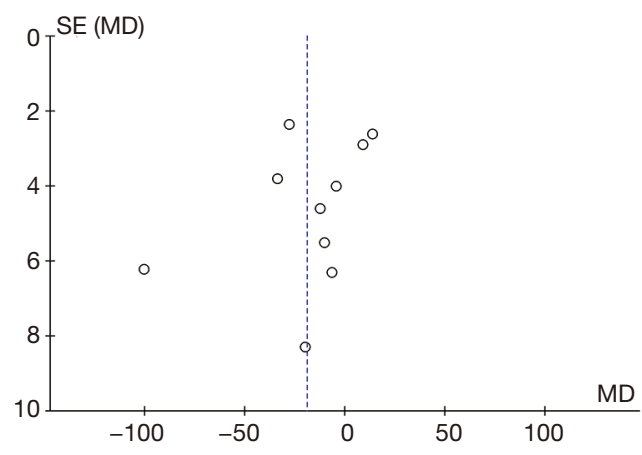

Figure 12 The funnel chart of aspartate transaminase levels from blockage of the hepatic hilum $(\mathrm{B}-\mathrm{HH})$ to time of opening of the hepatic hilum $(\mathrm{O}-\mathrm{HH})$.

\section{Acknowledgments}

Funding: None.

\section{Footnote}

Reporting Checklist: The authors have completed the PRISMA reporting checklist. Available at https://dx.doi. org/10.21037/apm-21-1242

Conflicts of Interest: All authors have completed the ICMJE uniform disclosure form (available at https://dx.doi. org/10.21037/apm-21-1242). The authors have no conflicts of interest to declare.

Ethical Statement: The authors are accountable for all aspects of the work in ensuring that questions related to the accuracy or integrity of any part of the work are appropriately investigated and resolved.
Open Access Statement: This is an Open Access article distributed in accordance with the Creative Commons Attribution-NonCommercial-NoDerivs 4.0 International License (CC BY-NC-ND 4.0), which permits the noncommercial replication and distribution of the article with the strict proviso that no changes or edits are made and the original work is properly cited (including links to both the formal publication through the relevant DOI and the license). See: https://creativecommons.org/licenses/by-nc-nd/4.0/.

\section{References}

1. Hausburg MA, Banton KL, Roman PE, et al. Effects of propofol on ischemia-reperfusion and traumatic brain injury. J Crit Care 2020;56:281-7.

2. Xu F, Ma R, Zhang G, et al. Estrogen and propofol combination therapy inhibits endoplasmic reticulum stress and remarkably attenuates cerebral ischemia-reperfusion injury and OGD injury in hippocampus. Biomed Pharmacother 2018;108:1596-606.

3. Yan HJ, Qi GQ, Ma Y. Effect of propofol on myocardial ischemia-reperfusion injury through MAPK/ERK pathway. Eur Rev Med Pharmacol Sci 2019;23:11051-61.

4. Wang B, Wu Q, Liao J, et al. Propofol Induces Cardioprotection Against Ischemia-Reperfusion Injury via Suppression of Transient Receptor Potential Vanilloid 4 Channel. Front Pharmacol 2019;10:1150.

5. Wei Q, Zhao J, Zhou X, et al. Propofol can suppress renal ischemia-reperfusion injury through the activation of PI3K/AKT/mTOR signal pathway. Gene 2019;708:14-20.

6. Li YM, Sun JG, Hu LH, et al. Propofol-mediated cardioprotection dependent of microRNA-451/HMGB1 against myocardial ischemia-reperfusion injury. J Cell 
Physiol 2019;234:23289-301.

7. Kim D, Choi JW, Han S, et al. Ischemic Preconditioning Protects Against Hepatic Ischemia-Reperfusion Injury Under Propofol Anesthesia in Rats. Transplant Proc 2020;52:2964-9.

8. Chang YC, Xue WJ, Ji W, et al. The Protective Effect of Propofol Against Ischemia-Reperfusion Injury in the Interlobar Arteries: Reduction of Abnormal Cx43 Expression as a Possible Mechanism. Kidney Blood Press Res 2018;43:1607-22.

9. Hao W, Zhao ZH, Meng QT, et al. Propofol protects against hepatic ischemia/reperfusion injury via miR-133a$5 \mathrm{p}$ regulating the expression of MAPK6. Cell Biol Int 2017;41:495-504.

10. Yu W, Gao D, Jin W, et al. Propofol Prevents Oxidative Stress by Decreasing the Ischemic Accumulation of Succinate in Focal Cerebral Ischemia-Reperfusion Injury. Neurochem Res 2018;43:420-9.

11. Yu X, Sun X, Zhao M, et al. Propofol attenuates myocardial ischemia reperfusion injury partly through inhibition of resident cardiac mast cell activation. Int Immunopharmacol 2018;54:267-74.

12. Liu Z, Meng Y, Miao Y, et al. Propofol reduces renal ischemia/reperfusion-induced acute lung injury by stimulating sirtuin 1 and inhibiting pyroptosis. Aging (Albany NY) 2020;13:865-76.

13. Zhang WY, Zhang QL, Xu MJ. Effects of propofol on myocardial ischemia reperfusion injury through inhibiting the JAK/STAT pathway. Eur Rev Med Pharmacol Sci 2019;23:6339-45.

14. Lotz C, Stumpner J, Smul TM. Sevoflurane as opposed to

Cite this article as: Feng C, Qian D, Chen C. A meta-analysis and systematic review of propofol on liver ischemia-reperfusion injury protection during hepatocellular carcinoma anesthesia surgery. Ann Palliat Med 2021;10(6):6726-6735. doi: 10.21037/ apm-21-1242 propofol anesthesia preserves mitochondrial function and alleviates myocardial ischemia/reperfusion injury. Biomed Pharmacother 2020;129:110417.

15. Fang H, Zhang FX, Li HF, et al. PRR34-AS1 overexpression promotes protection of propofol pretreatment against ischemia/reperfusion injury in a mouse model after total knee arthroplasty via blockade of the JAK1-dependent JAK-STAT signaling pathway. J Cell Physiol 2020;235:2545-56.

16. Sun B, Ou H, Ren F, et al. Propofol inhibited autophagy through $\mathrm{Ca} 2+/ \mathrm{CaMKK} \beta / \mathrm{AMPK} / \mathrm{mTOR}$ pathway in OGD/R-induced neuron injury. Mol Med 2018;24:58.

17. Leurcharusmee P, Sawaddiruk P, Punjasawadwong $Y$, et al. The Possible Pathophysiological Outcomes and Mechanisms of Tourniquet-Induced Ischemia-Reperfusion Injury during Total Knee Arthroplasty. Oxid Med Cell Longev 2018;2018:8087598.

18. Lin LN, Wang WT, Wu JZ, et al. Protective effect of propofol on liver during ischemia-reperfusion injury in patients undergoing liver surgery. Zhongguo Wei Zhong Bing Ji Jiu Yi Xue 2004;16:42-4.

19. Liu Z, Zhang J, Zhang F, et al. Propofol post-conditioning lessens renal ischemia/reperfusion-induced acute lung injury associated with autophagy and apoptosis through MAPK signals in rats. Gene 2020;741:144562.

20. Eroglu T, Bozkurt M, Kapi E, et al. A Study on the Effects of the Use of Propofol in Experimental Model Inferior Epigastric Island Flap on Ischemia-Reperfusion Injury. J Craniofac Surg 2017;28:2193-8.

(English Language Editor: J. Teoh) 